\title{
Technical note: Validation of an ear-tag accelerometer to identify feeding and activity behaviors of tiestall-housed dairy cattle
}

\author{
A. Zambelis, T. Wolfe, and E. Vasseur* \\ Department of Animal Science, McGill University, Ste-Anne-de-Bellevue, QC, Canada, H9X 3V9
}

\section{ABSTRACT}

The objective of this study was to validate the CowManager SensOor ear-tag accelerometer (Agis Automatisering BV, Harmelen, the Netherlands) against visual observations of feeding, rumination, resting, and active behaviors of tiestall-housed dairy cows. Prior validation of the sensor has been published for freestall and grazing dairy herds. However, the behavioral differences that exist among these and a tiestall system necessitate additional validation. Lactating Holstein cows $(\mathrm{n}=10)$ at different lactation stages and parities were included in the study. Cows were monitored both visually and with the sensor for $10 \mathrm{~h} / \mathrm{d}$ for 4 consecutive days ( 10 cows $\times 10 \mathrm{~h} \times 4 \mathrm{~d}=400 \mathrm{~h}$ of observation total). A single trained observer classified each minute of visual observation into 1 of 13 behaviors and then summarized them into the 4 behavioral categories of eating, rumination, not active, or active. The sensor registered ear movements continuously and, based on a proprietary model, converted them into the behavioral categories. Multivariate mixed models were run to obtain covariance estimates, from which correlation coefficients were computed to assess agreement between visual observation and sensor data. The models included the percentage of time spent performing each behavior per day as the dependent variable and technology (visual observation versus sensor) and day as fixed effects. The models also included the random effects of technology and the repeated effects of technology and day. The correlation strength between visual observation and sensor data varied from poor to almost perfect by behavioral category (eating: $\mathrm{r}=0.27$; rumination: $\mathrm{r}=0.69$; eating-rumination: $\mathrm{r}=0.83$; not active: $\mathrm{r}=$ 0.95 ; and active: $r=0.89$ ). The results suggest that the sensor can be used to accurately monitor active and not-active behaviors of tiestall-housed dairy cows. The results also suggest that although the sensor shows promise for identifying feeding behaviors in general,

Received September 28, 2018.

Accepted January 8, 2019.

*Corresponding author: elsa.vasseur@mcgill.ca the independent classification of rumination and eating requires additional sensitivity.

Key words: sensor, precision dairy technology, activity monitoring, feeding behavior, activity behavior

\section{Technical Note}

Activity monitors are becoming increasingly common on commercial dairies due to their ability to monitor key behavioral indicators, including heat detection, feed activity, and rumination. Precision dairy technologies have the potential to improve farm management and welfare monitoring through earlier detection of special care needs for individual animals (Vasseur, 2016). However, these technologies are based on automated measures of activity and behavior and therefore are dependent on the housing system in which the animals are kept. Most of the research on dairy cow comfort and welfare has focused on freestalls (Rushen et al., 2007) despite tiestalls remaining a predominant housing system in North America (e.g., $74.4 \%$ of dairy barns in Canada use a tiestall operation; Canadian Dairy Information Centre, 2016). Depending on whether behavioral expression occurs in tiestall versus loose-housing systems, its nature and extent varies due to limitations in space allowance and ability to move freely (Krohn, 1994). For instance, expression of social behaviors in a loose pen have been shown to be more frequent and antagonistic than those in a tiestall, where access to neighbors is limited and competition for resources is low (Krohn, 1994). Differences in the expression of behavior between housing systems can pose challenges for sensor accelerometers, which classify behavior based on the cow's acceleration in 3-dimensional space.

The CowManager SensOor ear-tag accelerometer (Agis Automatisering BV, Harmelen, the Netherlands) enables continuous monitoring of ear temperature, ruminating and eating behavior, and activity level of dairy cows. Ruminating and eating behaviors can been used to track individual feeding patterns and their links with illness risk and production measures (Diosdado et al., 2015). Fluctuations in activity level can indicate a range of physiological disturbances, including illness 
at lower levels and distress or estrus at higher levels (Diosdado et al., 2015). Continuous monitoring of these indicators allows small deviations in behavior to be used for earlier intervention efforts without greatly increasing management demands. For precision farming technologies to benefit researchers and producers, the accuracy and precision of the information being collected and reported must be validated. Although prior validation of the sensor has been published for freestall and grazing dairy herds (Bikker et al., 2014; Borchers et al., 2016; Pereira et al., 2018), the vast array of behavioral differences that exist among these and a tiestall system create the need for additional validation.

The objective of this study was to validate the CowManager SensOor ear-tag accelerometer against visual observations of feeding, rumination, resting, and active behaviors of tiestall-housed dairy cows. We hypothesized that the ability to validate discrete behaviors such as rumination, eating, and rest would be greater compared with active behaviors, which exist in numerous forms and may be dependent on the housing system (e.g., number of steps; Shepley et al., 2017).

During August 2017, lactating Holstein cows ( $\mathrm{n}=$ $10)$ in different lactation stages $(171.7 \pm 91.8 \mathrm{DIM})$ and parities $(3.4 \pm 1.1)$ were randomly selected from the McGill University Macdonald Campus Dairy Complex (Ste-Anne-de-Bellevue, QC, Canada). A minimum of 10 cows was required for a strong statistical evaluation of sensor versus visual observation agreement and yielded a correlation with 0.90 power, an $\alpha$-value of 0.05 , and an assumed effect size of 0.75 (correlation coefficient; Friedman, 1982). Cows were housed in a single row of tiestalls designed to fit each cow's size within $\pm 5.08 \mathrm{~cm}$ of current recommendation (DFC-NFACC, 2009). All stall bases were rubber mats (KKM longline; Distribution Multi-Mat Inc., Ste-Cécile-de-Milton, QC, Canada) coated with a small layer of sawdust, which was supplemented every morning. The stalls and gutters were scraped continuously by barn staff from 0500 $\mathrm{h}$ until $2100 \mathrm{~h}$. Cows were milked in-stall twice daily at a 12-h interval. Cows were fed a TMR 4 times daily, and feed was pushed closer to cows 6 times daily. Water was accessible ad libitum from a self-filling water bowl located between each pair of stalls.

Four video cameras (Smart Turret 2.8, Hikvision Digital Technology Co. Ltd., Hangzhou, China; 720p resolution at 8 frames/s) were positioned to each obtain a frontal view of 3 adjacent stalls (distance: 3.84 $\mathrm{m}$, height: $1.98 \mathrm{~m}$ from center stall). Cows were video recorded continuously for 5 consecutive days. Twelve hours before the start of video recording, cows were equipped with a CowManager SensOor ear-tag sensor. The molded sensor was mounted on the left ear of each cow into a regular radio-frequency identification-type electronic tag recognized by the Canadian Food Inspection Agency. Three-dimensional movement of each cow's ear was registered continuously by an accelerometer within the sensor. A wireless router collected the data from the sensors and transmitted the information to a coordinator connected to a computer to ensure proper data flow. Based on a private model, the sensorregistered ear movements were converted into the 5 behavioral categories of eating, rumination, not active, active, and high active. These data were collected and presented by a web application as the percentage of each behavioral category on a per-hour or per-day basis. The active and high active categories were summed to obtain a total measure of activity because estrusrelated behaviors were not considered separately from other active behaviors.

Video recording was used to assess agreement of behavioral classification of the sensor versus visual observation during preselected times. The first $24 \mathrm{~h}$ of video recording was used as a prevalidation trial to define five 2-h blocks, each block selected as a peak period of activity for 1 of the 4 behavioral categories. The 5 selected 2-h periods were 0100 to $0300 \mathrm{~h}, 0800$ to $1000 \mathrm{~h}, 1000$ to $1200 \mathrm{~h}, 1400$ to $1600 \mathrm{~h}$, and 1900 to $2100 \mathrm{~h}$, amounting to $10 \mathrm{~h}$ of video recording per cow for 4 consecutive days ( $400 \mathrm{~h}$ total). These blocks were scored on a per-minute basis by a single trained observer, who assigned a score from 0 to $4(0: 0 \mathrm{~s}, 1$ : 1-5 s, 2: 6-10 s, 3: 11-30 s, 4: 31-60 s) to each of the 12 behaviors outlined in Table 1 based on their duration. Each of the behaviors was then reclassified into a condensed category to allow for direct comparison with the 4 behavioral categories (Table 1). Each minute of video recording was then defined as the primary condensed category observed. The condensed categories were then presented as a percentage of activity per hour. To measure consistency of scoring across time, intraobserver reliability was tested at the start of the trial and again after scoring each cow. Intraobserver reliability was evaluated by comparing the per-minute condensed category scores from $10 \mathrm{~h}$ of pretrial video recording of a single cow. All the measures had almost perfect agreement, with an average ( \pm SD) Cohen's (1960) kappa coefficient of $0.96 \pm 0.03$ for eating, 0.98 \pm 0.01 for rumination, $0.96 \pm 0.03$ for resting, and 0.89 \pm 0.08 for active (following the system of Landis and Koch, 1977).

Agreement between visual observation and sensor data was evaluated using the percentage of time each behavior was performed per day. An additional behavioral category for eating-rumination was created by summing the percentage of time spent ruminating and eating for each hour and then combining hours to yield a percentage per day. The distribution of the data was 
Table 1. Ethogram categorizing and defining the visually observed behaviors of tiestall-housed dairy cattle ${ }^{1}$

\begin{tabular}{lll}
\hline Behavioral item & Behavioral category & Behavior description \\
\hline Eating $^{2}$ & Eating & The muzzle is located in or above the feed, with chewing or licking movements \\
Rumination $^{2}$ & Rumination & Rhythmic side-to-side chewing of cud \\
Resting $^{2}$ & Resting & Lying or standing idly with no behavioral modifier \\
Exploring $^{2}$ & Active & Smelling, touching, licking, or seeking of any material, floor, or infrastructure \\
Drinking & Active & The muzzle is located in the water bowl, with ingestion of fluids \\
Urination & Active & Elimination of urine from the body \\
Defecation & Active & Elimination of feces from the body \\
Rising & Active & Transition in posture from lying to standing \\
Lying down & Active & Transition in posture from standing to lying \\
Head swing $^{2}$ & Active & Forcefully tossing the head rotationally toward the body \\
Autogrooming & Active & Licking or rubbing own head or body surface with self or infrastructure \\
Association $^{2}$ & Active & Initiator or recipient of agonistic or nonagonistic social interactions \\
\hline
\end{tabular}

${ }^{1}$ Adapted from Bikker et al. (2014).

${ }^{2}$ Behavioral item can occur for both a lying posture and a standing posture.

first assessed to confirm normality. A 2-sided paired $t$-test was then used to compare visual observation and sensor agreement on the percentage of total time spent by each cow performing the behaviors. All data were analyzed using SAS 9.4 (SAS Institute Inc., Cary, NC).

An alternate statistical approach was then used to measure the relationship between visual observation and sensor data while accounting for the intricate design structure and repeated measures nature of the data. Multivariate mixed models were run to obtain covariance parameter estimates. The models included the percentage of time spent performing each behavior per hour as the dependent variable and technology (sensor vs. visual observation) and day as fixed effects. The models also included the random effect of technology and the repeated effects of technology and day. The covariance parameter estimates produced by the models were then used to calculate correlation coefficients between sensor and visual observation data with the equations presented by Dimassi (2008). Pearson correlation coefficients were characterized according to the criteria of Hinkle et al. (2003) as being negligible (0.00-0.30), low (0.30-0.50), moderate (0.50-0.70), high (0.70-0.90), or very high (0.90-1.00).

The percentage of total time spent performing each behavior and the analysis of sensor and visual observation agreement are presented in Table 2. The percentage of total time spent ruminating by each cow was found to be 9 percentage units higher $(P<0.0001)$ for the sensor compared with visual observation. The proportion of total time spent eating by each cow was recorded by the sensor to be 10.6 percentage units lower $(P<0.0001)$ than that recorded by visual observation. No difference was found between sensor and visual observation for the percentage of total time each cow was active $(23.4 \%$ vs. $22.1 \% ; P=0.20)$.

The correlation between sensor and visual observation for percentage of time spent eating was negligible $(\mathrm{r}=0.27 ; P=0.18)$. For percentage of time spent ruminating, the correlation between sensor and visual observation was moderate $(\mathrm{r}=0.69 ; P<0.0001)$. The correlation found for eating differs from prior validation studies conducted in freestall and grazing dairy herds, where this correlation was found to be consistently high at 0.88 (Bikker et al., 2014; Borchers et al., 2016; Pereira et al., 2018). In contrast, previous studies have reported inconsistent correlations between sensor and visual observation for rumination even within a single housing system (e.g., freestall barn), ranging from moderate $(r=0.69$; Borchers et al., 2016) to high $(r=0.93$; Bikker et al., 2014). Pereira et al. (2018) yielded results similar to those of Borchers et al. (2016) despite conducting their study in a grazing dairy herd $(\mathrm{r}=0.72)$. Both Borchers et al. (2016) and Pereira et al. (2018) attributed these moderate correlations to a difficulty in observer ability to consistently and accurately record

Table 2. Percentage of total recorded time spent performing each behavior ( $\pm \mathrm{SD}$ ) for sensor versus visual observation with $P$-values for the 2-sided paired $t$-test, and Pearson correlation coefficient $(\mathrm{r})$ with $P$-values for analyzing the strength of the relationship between sensor and visual observation data

\begin{tabular}{|c|c|c|c|c|c|}
\hline Behavior category & Sensor & Visual observation & $P$-value for $t$-test & $\mathrm{r}$ & $P$-value for $\mathrm{r}$ \\
\hline Eating & $6.1 \pm 0.03$ & $16.7 \pm 0.04$ & $<0.0001$ & 0.27 & 0.18 \\
\hline Resting & $31.6 \pm 0.06$ & $30.7 \pm 0.06$ & 0.08 & 0.95 & $<0.0001$ \\
\hline Eating-rumination & $45.2 \pm 0.05$ & $47.1 \pm 0.05$ & 0.08 & 0.83 & $<0.0001$ \\
\hline
\end{tabular}


rumination due to inadequate views of cows mingling within the herd. To avoid the same issue, we designed our study with a pretested camera placement that would ensure a continuous nonobstructed frontal view of all behaviors. To facilitate accurate and consistent scoring, a detailed ethogram was also created to ensure predefined and established criteria for classification.

We therefore postulate that the moderate correlation found was due to the nature of behavior conducted in a tiestall and the limitation of the technology to sensitively record rumination. In a freestall, eating and rumination occur in separate and distinct areas of the pen, with eating behavior occurring on pasture or in a feed bunk and rumination occurring in the alleys and stalls. In a tiestall system, however, rumination and eating occur in rapid succession at the stall, with head placement in proximity to the feed being the main source of variation. Because both rumination and eating involve rhythmic side-to-side chewing, the accelerometer within the sensor may have difficulty distinguishing between minor differences in ear acceleration when the behaviors occur in rapid succession to one another. It is also possible that the pattern of ear motion associated with ruminating and eating behaviors varies between tiestall and loose-housing systems. To further assess this possibility, time budgets of the behaviors as estimated by the sensor were compared with prior literature.

According to the sensor, eating behavior represented an average of $5.6 \pm 4.1 \%$ of daily activity, equivalent to $1.4 \mathrm{~h} / \mathrm{d}(0.056 \times 24 \mathrm{~h} / \mathrm{d}=1.4 \mathrm{~h} / \mathrm{d})$ for each cow. This finding is distinctly lower than the expected average daily mealtime of a lactating Holstein dairy cow, as apparent in prior research. Gomez and Cook (2010) used continuous video recording to measure the time budgets of lactating dairy cattle $(\mathrm{n}=205)$ and found that average time feeding was $4.3 \pm 1.1 \mathrm{~h} / \mathrm{d}$. Even if only considering the lower quartile of these cows, average time feeding was still $3.5 \mathrm{~h} / \mathrm{d}$, more than 2 times greater than that detected by the sensor. In the present study, the average time spent eating was measured across the total recorded time using visual observation only, so a direct daily comparison cannot be made. However, total eating time constituted an average of $16.7 \pm 0.04 \%$ of all activity in the time considered, equivalent to 4.01 $\mathrm{h} / \mathrm{d}$ if extrapolated across the day. This not only is similar to the average daily eating time reported by Gomez and Cook (2010) but also is comparable with that reported by Bikker et al. (2014; visual: $13.0 \pm$ 4.94; sensor: $15.9 \pm 5.02$ ), wherein strong agreement was found between sensor and visual observation for eating time. Based on this evidence, the sensor seems prone to underestimating the percentage of eating time performed by tiestall-housed dairy cattle.
Because the sensor classifies activity on a continuous basis, the underestimation of eating time must have instead been classified as one of the other behavioral categories. Although sensor estimations were higher than those by visual observation for all other categories, the difference in the percentage of total time spent ruminating by each cow was by far the most significant (39.1\% vs. $30.4 \% ; P<0.0001)$. All other behavioral categories had high to very high agreement between sensor and visual observation and so could not singularly account for the large underestimation of eating time. Because the online application for the sensor reports behavior data in hourly or daily summaries with no access to the raw data, it was not possible to conduct a direct comparison of the disagreements. Instead, the category of eating-rumination was created by pooling rumination and eating data together to assess the sensor's ability to monitor feeding-related behaviors without the additional sensitivity required for discrimination of rumination and eating at the stall. Thus, the correlation between sensor and visual observation for the measure of eating-rumination was high $(\mathrm{r}=0.83 ; P<0.0001)$, demonstrating a strong ability by the sensor to monitor feeding-related behaviors in a tiestall system.

The correlation between sensor and visual observation for percentage of resting time was very high ( $\mathrm{r}$ $=0.95 ; P<0.0001)$. A very high correlation of 0.98 was also found by Bikker et al. (2014), demonstrating the sensor's ability to monitor not-active behavior in both freestall and tiestall dairy herds. It is not surprising that both studies found similar results because regardless of housing type, resting behavior comprises the absence of movement in both standing and lying positions. Because the sensor uses an accelerometer to measure changes in the cow's ear position to identify discrete forms of behavior, a lack of movement is likely the simplest to detect. In contrast to the original hypothesis, the correlation between sensor and visual observation for percentage of time spent active was high $(\mathrm{r}=0.89 ; P<0.0001)$. This finding differs from weaker correlations found by Bikker et al. (2014; $\mathrm{r}=$ $0.73)$ and Pereira et al. $(2018 ; \mathrm{r}=0.20)$. In accordance with our original predictions, these studies suggested that active behaviors are more difficult to define and categorize for both the observer and sensor due to their complexity. Based on these challenges, we designed our study with a pretested camera placement and detailed ethogram to ensure nonobstructed viewing and consistent classification criteria for all behaviors. As such, we postulate that the ability of both sensor and observer to accurately monitor activity in a tiestall is improved due to the lower complexity and variation of movement possible in the restricted space. This is not to say that 
total exploratory behavior is reduced in the tiestall; the contrary was found by Krohn (1994), who reported that the frequency of all types of exploratory behavior recorded was 2 to 3 times higher in the tiestall than in the loose-housing system. Instead, tiestalls reduce freedom of movement, influencing the range and complexity of motion possible (Krohn, 1994). Consequently, most of the active behaviors observed were simple movements related to biological necessity (e.g., urination, defecation, changes in posture, drinking, autogrooming) and only a few encompassed complex interactions with stimuli (e.g., association and exploring). Despite original predictions, the ability to validate rumination and eating was not greater than the ability to validate active behaviors due to the dependency of all of these behaviors on the housing system in which they occur. For these reasons, the sensor demonstrated a strong ability to monitor active behavior in a tiestall system.

We believe this to be the first validation study comparing visual observation against CowManager sensor data for a tiestall dairy herd. The results suggest that the sensor can be used to accurately monitor active and not-active behaviors of tiestall-housed dairy cows for both scientific and management purposes. The results also suggest that although the sensor shows promise for identifying eating-rumination, the independent classification of rumination and eating requires additional sensitivity. We believe that to optimize behavior detection and reduce inconsistencies across validation studies, precision technologies based on automated measures for activity must be developed and validated for specific housing systems.

\section{ACKNOWLEDGMENTS}

We acknowledge the support of our funding partners, the Natural Sciences and Engineering Research Council of Canada (Ottawa, ON, Canada), Novalait (Quebec City, QC, Canada), Dairy Farmers of Canada (Ottawa, ON, Canada), and Valacta (Sainte-Anne-de-Bellevue, QC, Canada) through Vasseur's Industrial Research Chair. A big thank-you goes to Roger I. Cue for his guidance and expertise on planning the statistical analysis and project design. We also extend our thanks to the staff of the McGill University Macdonald Campus Dairy Complex (Ste-Anne-de-Bellevue, QC, Canada).

\section{REFERENCES}

Bikker, J. P., H. Van Laar, P. Rump, J. Doorenbos, K. Van Meurs, G. M. Griffioen, and J. Dijkstra. 2014. Evaluation of an ear-attached movement sensor to record cow feeding behavior and activity. J. Dairy Sci. 97:2974-2979. https://doi.org/10.3168/jds.2013-7560.

Borchers, M. R., Y. M. Chang, I. C. Tsai, B. A. Wadsworth, and J. M. Bewley. 2016. A validation of technologies monitoring dairy cow feeding, ruminating, and lying behaviors. J. Dairy Sci. 99:74587466. https://doi.org/10.3168/jds.2015-10843.

Canadian Dairy Information Centre. 2016. The farm: Types of dairy barns. Accessed Apr. 5, 2018. http://www.dairyinfo.gc.ca/index_e .php?s1=dff-fcil\&s2=farm-ferme\&s3=db-el.

Cohen, J. 1960. A coefficient of agreement for nominal scales. Educ. Psychol. Meas. 20:37-46. https://doi.org/10.1177/ 001316446002000104.

DFC-NFACC (Dairy Farmers of Canada-National Farm Animal Care Council). 2009. Code of Practice for the Care and Handling of Dairy Cattle. Dairy Farmers of Canada and the National Farm Animal Care Council, Ottawa, ON, Canada.

Dimassi, O. 2008. Correlation in repeated measures designs. Accessed Sep. 6, 2018. https://www.uni-hohenheim.de/bioinformatik/ beratung/fallstudien/Correlation\%20in\%20repeated\%20measures $\%$ 20designs.doc.

Diosdado, J. A. V., Z. E. Barker, H. R. Hodges, J. R. Amory, D. P. Croft, N. J. Bell, and E. A. Codling. 2015. Classification of behaviour in housed dairy cows using an accelerometer-based activity monitoring system. Anim. Biotelem. 3:15. https://doi.org/10 .1186/s40317-015-0045-8.

Friedman, H. 1982. Simplified determinations of statistical power, magnitude of effect and research sample sizes. Educ. Psychol. Meas. 42:521-526. https://doi.org/10.1177/001316448204200214.

Gomez, A., and N. B. Cook. 2010. Time budgets of lactating dairy cattle in commercial freestall herds. J. Dairy Sci. 93:5772-5781. https://doi.org/10.3168/jds.2010-3436.

Hinkle, D. E., W. Wiersma, and S. G. Jurs. 2003. Applied Statistics for the Behavioral Sciences. 5th ed. Houghton Mifflin, Boston, MA.

Krohn, C. C. 1994. Behaviour of dairy cows kept in extensive (loose housing/pasture) or intensive (tie-stall) environments. III. Grooming, exploration and abnormal behaviour. Appl. Anim. Behav. Sci. 42:73-86. https://doi.org/10.1016/0168-1591(94)90148-1.

Landis, J. R., and G. G. Koch. 1977. The measurement of observer agreement for categorical data. Biometrics 33:159-174. https://doi .org/10.2307/2529310.

Pereira, G. M., B. J. Heins, and M. I. Endres. 2018. Validation of an ear-tag accelerometer sensor to determine rumination, eating, and activity behaviors of grazing dairy cattle. J. Dairy Sci. 101:24922495. https://doi.org/10.3168/jds.2016-12534.

Rushen, J., D. Haley, and A. M. De Passillé. 2007. Effect of softer flooring in tie-stalls on resting behavior and leg injuries of lactating cows. J. Dairy Sci. 90:3647-3651. https://doi.org/10.3168/jds .2006-463.

Shepley, E., M. Berthelot, and E. Vasseur. 2017. Validation of the ability of a 3D pedometer to accurately determine the number of steps taken by dairy cows when housed in tie-stalls. Agriculture 7:53. https://doi.org/10.3390/agriculture7070053.

Vasseur, E. 2016. Optimizing outcome measures of welfare in dairy cattle assessment. J. Anim. Sci. 94(Suppl. 5):45. (Abstr.) https:// doi.org/10.2527/jam2016-0097. 